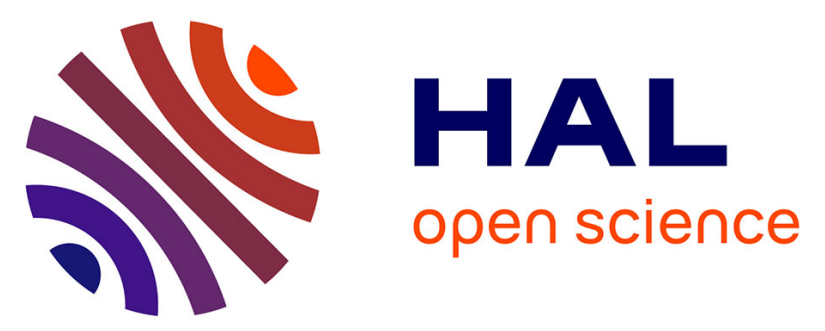

\title{
L'indifférence des Français et des Belges (francophones) pour leurs voisins Européens: une pièce de plus au dossier de l'absence de communauté politique européenne?
}

Sophie Duchesne, Virginie van Ingelgom

\section{To cite this version:}

Sophie Duchesne, Virginie van Ingelgom. L'indifférence des Français et des Belges (francophones) pour leurs voisins Européens: une pièce de plus au dossier de l'absence de communauté politique européenne?. Politique européenne, 2008, 3 (26), pp.143-164. 10.3917/poeu.026.0143 . halshs00841656

\section{HAL Id: halshs-00841656 \\ https://shs.hal.science/halshs-00841656}

Submitted on 5 Jul 2013

HAL is a multi-disciplinary open access archive for the deposit and dissemination of scientific research documents, whether they are published or not. The documents may come from teaching and research institutions in France or abroad, or from public or private research centers.
L'archive ouverte pluridisciplinaire HAL, est destinée au dépôt et à la diffusion de documents scientifiques de niveau recherche, publiés ou non, émanant des établissements d'enseignement et de recherche français ou étrangers, des laboratoires publics ou privés.

\section{(1)(1) $\$(0)$}

Distributed under a Creative Commons Attribution - NonCommercial - ShareAlikel 4.0 


\title{
L'indifférence des Français et des Belges (francophones) pour leurs voisins Européens : une pièce de plus au dossier de l'absence de communauté politique européenne?
}

\author{
Sophie Duchesne (CNRS/Sciences Po). sophie.duchesne@ @sciences-po.fr \\ Virginie Van Ingelgom (F.R.S.-FNRS/UCL-SPRI). vaningelgom@spri.ucl.ac.be
}

Version avant travail de l'éditeur du texte publié dans Politique Européenne, décembre 2008

Résumé:

Partant du constat d'une asymétrie entre la mise en place d'une citoyenneté européenne et la persistance du cadre d'identification nationale des Européens, ce texte aborde la question des sentiments réciproques que se portent les Européens dans le cadre d'une interrogation sur les mécanismes de création d'une communauté politique européenne : est-ce qu'une «préférence pour les autres Européens » pourrait, dans le moyen terme, compenser l'absence de connaissance, d'intérêt et d'attachement des Européens pour leur Union ? L'analyse porte sur 8 focus groups réalisés en Belgique (francophone) et en France, selon une méthodologie particulière qui vise à faciliter l'expression des objets de conflit entre participants ; ces discussions sont par là-même particulièrement marquées par les émotions. Les émotions manifestées par les participants à ces groupes à l'égard des autres Européens sont analysées grâce à la combinaison de trois méthodes aussi différentes que complémentaires (analyse interprétative de la dynamique des groupes, analyse automatique de texte et codification). L'analyse montre que si les Européens - et plus particulièrement les Européens de l'Ouest -, sont bien présents dans ces discussions, l'intensité émotionnelle des échanges qui portent sur eux est faible et ne permet pas de nourrir la thèse de la préférence européenne. Par contre, elle suggère qu'ils ne possèdent pas (plus ?) à l'égard des Belges (francophones) et des Français la qualité «d'autre », au sens de ceux qui fondent l'identité de soi par la différence. Un résultat qui prendra un sens différent pour les tenants du post-nationalisme et ceux qui voient dans l'intégration européenne un processus similaire aux constructions nationales des siècles passés.

\section{French and (French speaking) Belgian lack of interest in their European neighbours: another piece of evidence for the case of the missing European political community?}

Summary:

Starting from the asymmetry observed between the setting up of a European citizenship and the persistent national identifications of Europeans, this paper addresses the question of the reciprocal feelings of Europeans for each other within the framework of a broader question about mechanisms for the formation of a European political community: in the middle term, could some "preference for other Europeans" compensate for the fact that Europeans have little knowledge of, interest in or attachment to their Union? The analysis is based on results from eight focus groups organized in Paris and Brussels using a moderation technique to foster the expression of disagreement between participants; because they are conflictive, these discussions are characterised by their emotionality. We analyse emotions shown by participants regarding other Europeans thanks to three different but complementary techniques (interpretative analysis of the discussion dynamics; automatic content analysis; hand-made codification). The analysis shows that if Europeans - and more specifically Western Europeans - are indeed discussed, this happens without much display of emotion thereby limiting support for the thesis of European preference. However, this does suggest 
that, as far as the French and the (French speaking) Belgians are concerned, Western Europeans do not (no longer?), belong to the "others" category, in the sense of people who contribute to the self-definition, the making of "us", by differentiation. This result will be interpreted differently by post-nationalists and by those who consider European integration to be a process similar to nation-building in past centuries.

La notion de communauté politique est une notion complexe, du fait de la diversité des formes qu'elle peut ou a pu emprunter dans l'histoire. En démocratie, et plus précisément, dans les démocraties occidentales qui se sont développées depuis le $18^{\text {ème }}$ siècle, qui dit communauté politique dit citoyens mais aussi, le plus souvent, nationaux. Les membres des communautés politiques formées par les Etats-nations démocratiques d'Europe en particulier, sont historiquement à la fois ceux qui possèdent la nationalité et jouissent des droits civiques. Sur le plan théorique, le recouvrement des statuts de national et de citoyen a fait et fait toujours l'objet de nombreux débats ${ }^{1}$. Par contre, la sociologie historique a bien montré comment la «fabrique» des citoyens, i.e. l'apprentissage des normes et des comportements sur lesquels reposent le fonctionnement de la démocratie de masse s'est nourrie du développement parallèle des nations et de ce qu'on a coutume de désigner par «identité nationale » (Déloye, 1996). En écho au discours de la raison et de l'universel qui fonde les notions de bien commun et de droits à l'aune desquelles on évalue les performances du «bon citoyen », le discours patriotique, chantre de la souveraineté nationale, a lui privilégié les sentiments liés à l'appartenance: fierté et fraternité. Autrement dit, la notion de communauté politique (nationale) telle que nous la connaissons à la fin du $20^{\text {eme }}$ siècle dans les pays de l'Union européenne mêle de façon intrinsèque raison et sentiments.

Au même moment, la poursuite de l'intégration européenne a amené ses promoteurs à souhaiter le dépassement d'une logique d'union économique dans une volonté de création d'une communauté politique européenne. Ce projet a notamment été affiché, dans le Traité de Maastricht, par l'institution d'une citoyenneté de l'Union. Par contre, la «nationalité européenne » n'a pas été créée : les citoyens européens sont les nationaux des pays de l'Union. La communauté politique européenne rompt donc avec la symétrie historique des communautés politiques nationales (Déloye, 2006). Le thème du déficit démocratique illustre l'échec relatif de cette construction, que manifestent la faiblesse des taux de participation aux élections européennes ainsi que l'usage très limité que les citoyens de l'Union font des droits qui leur ont été ouverts (Strudel, 2002). L'échec des référendums de ratification du Traité constitutionnel puis du Traité de Lisbonne est venu confirmer, pour nombre de commentateurs politiques, l'absence d'attachement des citoyens à leur Union ${ }^{2}$, par ailleurs très largement documentée par les analystes des données Eurobaromètres (Citrin \& Sides, 2004).

On sait que l'Union européenne, en tant que système politique, demeure un objet lointain, mal connu des citoyens, et que cette distance est de fait peu propice au

\footnotetext{
${ }^{1}$ David Miller par exemple est un ardent défenseur de la limitation de la citoyenneté au cadre national (Miller, 2002) contre des gens qui, comme Yasmine Soysal ou Jürgen Habermas, pensent eux la citoyenneté dans le dépassement du cadre national (Soysal, 1994 ; Habermas, 2000)

${ }^{2}$ Même si l'analyse détaillée des résultats montre que les motivations de ce vote ne permettent pas de l'assimiler simplement à un vote eurosceptique comme le montrent par exemple Nicolas Sauger, Sylvain Brouard et Emiliano Grossman pour le référendum français de 2005 (Sauger, Brouard \& Grossman, 2007).
} 
développement d'une relation affective entre les citoyens et le système politique européen ${ }^{3}$. $\mathrm{Ne}$ faut-il pas alors aller plutôt chercher les éléments affectifs du côté des nations européennes, autrement dit, regarder si la poursuite de l'intégration se manifeste par le développement d'un attachement à l'égard des autres nations européennes ? On peut supposer que le développement d'une forme de "préférence pour les autres Européens » pourrait compenser, ne serait-ce que provisoirement, le déficit d'attachement des citoyens à l'Europe, et nourrir en sentiments le rôle de citoyen de l'Union (Delmotte, 2007). Transformer les sentiments négatifs que les Européens entretenaient à l'égard des uns les autres en sentiments positifs, n'est-ce pas ce qu'avaient en tête, au sortir de la guerre, les «Pères Fondateurs » de l'Europe ?

\section{Hypothèse de départ : les Européens de l'Ouest dans «l'angle mort» des représentations nationales des Français et des Belges (francophones).}

Nous allons tenter, dans cet article, d'identifier les sentiments que les Français et les Belges (francophones) entretiennent vis-à-vis des autres Européens. Nous allons étudier, à l'aide d'entretiens collectifs ${ }^{4}$, la façon dont nos participants en parlent, en centrant l'analyse sur la teneur affective de ces propos, analyse qui combinera plusieurs méthodes. Les données dont nous disposons sont particulièrement appropriées à l'exercice. Il s'agit de huit focus groups, réalisés entre décembre 2006 et février 2007, rassemblant des participants choisis pour leur homogénéité sociale et leur hétérogénéité politique ${ }^{5}$, et représentant chacun trois heures environ de discussion sur l'Europe. Le scénario de discussion ${ }^{6}$ ainsi que la méthode d'animation des groupes ont été conçus pour rendre possible, voire favoriser la conflictualisation de la discussion. Or qui dit conflit, dit émotions. On sait en effet que l'ordre social ne favorise pas les comportements conflictuels, notamment dans leur rapport à la politique (Hamidi, 2006) - en tout cas, pas dans un espace public tel que nous l'avons construit. Mais nous avons organisé les conditions de possibilité de tels comportements en jouant sur l'ordre des questions, la composition des groupes réunissant des participants aux vues opposées (en tout cas sur papier), la longueur des débats, l'inclusion d'un temps de sociabilité (repas), la non-directivité de l'animation, l'affichage au fur et à mesure des idées exprimées mettant en évidence les ambivalences et les contradictions, ainsi que l'usage valorisé d'un marqueur de désaccord ${ }^{7}$.

\footnotetext{
${ }^{3} \mathrm{Au}$ point que ces sentiments ont surtout été pris en compte de manière négative dans la question du développement d'une identité européenne, à travers l'hypothèse, pourtant très tôt statistiquement mise en cause, d'un antagonisme entre identités nationale et européenne (Duchesne \& Frognier, 2008).

${ }^{4}$ Cette enquête, réalisée en collaboration avec Florence Haegel (Cevipof/Sciences-Po), André-Paul Frognier (SPRI, Université Catholique de Louvain), Elizabeth Frazer (New College et Department of Politics and International Relations, University of Oxford) et Guillaume Garcia (Centre Européen de Sciences-Po), a fait l'objet d'une présentation dans un précédent numéro de cette revue (Duchesne \& Van Ingelgom, 2008). Cet article ne traite que des focus groups réalisés à Paris et Bruxelles. Sur le choix des terrains, le lecteur peut se référer à l'article de Politique européenne précité. Merci à Olivier Rozenberg, ainsi qu'aux éditeurs de ce numéro, pour leurs commentaires.

${ }^{5}$ Quatre catégories de groupes ont été organisées : classes populaires, professions intermédiaires et cadres, ainsi qu'une catégorie témoin de militants de partis. Sur le plan politique, nous avons volontairement sélectionné des gens dont la préférence politique et les attitudes à l'égard de l'intégration divergeaient. Pour le détail du recrutement, voir (Garcia et alii, 2008).

${ }^{6}$ «Etre européen, qu'est-ce que ça veut dire ? Comment répartir le pouvoir en Europe ? A qui profite l'Europe ? Pour ou contre l'entrée de la Turquie dans l'UE ? Quelle est la position des partis politiques nationaux sur cette question?»

${ }^{7}$ La technique dite «de l'éclair » consiste à annoncer dès le début, comme une règle du jeu, qu'on attend des participants qu'ils réagissent s'ils voient affiché un propos avec lequel ils ont le moindre désaccord. L'animatrice reçoit une demande «d'éclair » avec enthousiasme, l'inscrit sur le tableau, où elle affiche au fur et à mesure ce
} 
De fait, ces discussions font une part plus importante aux émotions que des entretiens classiques. Elles sont donc propices à une analyse des sentiments que les participants associent aux objets dont ils traitent, et notamment, pour ce qui nous intéresse ici, aux pays qu'ils évoquent au fil des débats. Elles supposent dès lors une analyse appropriée. La première étape a consisté en un travail d'interprétation à partir des enregistrements. Celle-ci porte essentiellement sur la dynamique de la discussion, et sur les objets et les thèmes à partir desquels les participants s'impliquent réellement dans la discussion, autrement dit, à partir desquels ils éprouvent le besoin de faire connaître leur opinion, quitte à courir le risque d'un conflit avec les autres participants. Cette prise en compte de la conflictualité des interactions constitue en quelque sorte un marqueur de saillance des opinions, sur un sujet - l'UE - pour lequel on peut craindre que les opinions exprimées soient peu fondées (Duchesne \& alii, 2008).

De cette première analyse, il ressort d'abord de manière générale que les différences nationales de «framing » restent déterminantes, les participants s'appropriant l'UE à partir de catégories de compréhensions tirées de leur système politique national (Haegel \& alii, 2008). Les différences sociales sont également fortes, mais portent sur la distance entretenue à l'égard d'un système politique vécu surtout comme un renforcement de la domination, politique et économique. Par delà ces différences, une part essentielle des échanges, évaluée en terme d'intensité, porte sur des considérations d'ordre économique, en rapport avec la globalisation. L'analyse interprétative conduit à mettre en cause l'existence d'une catégorie «pays européens », dès lors que les participants parlent très différemment des Européens de l'Ouest et des Européens de l'Est. Les premiers ne font pas l'objet de fortes implications de la part des participants, contrairement aux Européens de l'Est d'une part, et à d'autres pays, notamment la Chine et les Etats-Unis. Au point que nous faisons l'hypothèse que les Européens de l'Ouest sont, en quelque sorte, pour les Français et les Belges (francophones), entrés dans ce que nous désignerons comme un «angle mort» de leur système de représentations des autres nationalités: ils ne sont plus vraiment des «autres ${ }^{8}$ et pas (encore ?) des «nous».

Nous aurions pu développer cette hypothèse en nous appuyant uniquement sur des extraits sélectionnés en fonction de nos interprétations. Mais nous avons voulu la mettre à l'épreuve avec un traitement plus systématique du matériau. Pour ce faire, nous avons utilisé deux outils : un logiciels de traitement automatique du discours, le logiciel Alceste; un logiciel d'aide à la codification, Atlas.ti. Le premier va nous aider à préciser la place que les Européens de l'Ouest occupent dans les discussions que nous avons organisées ; le second, à systématiser l'analyse des affects attachés à leurs évocations.

\section{Bref aperçu du corpus : un univers de signification de la nationalité centré sur la comparaison Europe de l'Ouest/Amérique et marqué par l'affectivité.}

Le logiciel Alceste fonctionne sur la base des cooccurrences et produit une classification des segments de texte décrite à travers les mots qui caractérisent chaque classe, chacune considérée comme un « univers sémantique » (Reinert, 1993 ; Brugidou, 2008). Cette analyse

qui se dit, à l'aide d'un éclair rouge, et s'assure que cet éclair fera par la suite l'objet d'une discussion spécifique (Duchesne \& Haegel, 2004, 883).

${ }^{8}$ En référence aux travaux classiques de psychologie sociale qui montrent comment le sentiment d'appartenance à un groupe social est notamment le produit de la construction d'une frontière, d'une différence avec les membres extérieurs au groupe, entre le «nous » et le « eux » (Tajfel, 1982). 
va nous permettre de présenter rapidement le contenu des discussions et d'évaluer la part relative faite aux évocations des Européens et plus largement, des nationalités dans le corpus. L'analyse Alceste ${ }^{9}$ réalisée sur les corpus belge et français (soit un document de 271053 mots) produit six classes ${ }^{10}$ d'unités élémentaires de texte, de tailles différentes. Elles sont caractérisées par six ensembles de mots significativement surreprésentés dans ces classes (tableau 1).

Tableau 1 : Formes très caractéristiques des six classes

\begin{tabular}{|l|l|}
\hline A. Nations & $\begin{array}{l}\text { américain(21), militaire(12), bas(20), guerre(46), pays(134), puissance(14), } \\
\text { union(40), économie(75), aujourd'hui(44), armée(13), partie(39), Amérique(16), } \\
\text { Etats-Unis(32), Tunisie(8), sentir(20), anglais(18), asiatique(8), Russie(11), } \\
\text { blocs(7), histoire(33), lieu(6), sentiment(10), rentrer(25), catastrophe(6), chimère(6), } \\
\text { différent(50), Yougoslavie(9), continent(7), haut(12), né(8), positif(8), social(27), } \\
\text { Allemagne(17) }\end{array}$ \\
\hline B. Discussion & $\begin{array}{l}\text { carte(44), discussion(41), éclair(44), idée(69), minute(12), objection(9), parole(19), } \\
\text { question(87), règle(26), tableau(22), aller(202), discuter(30), écrire(20), } \\
\text { enregistrer(10), finir(22), mettre(82), noter(14), passer(31), réfléchir(24), } \\
\text { répondre(15), écrit(20), gommette(26), suivant(15), ensemble(19), façon(17), } \\
\text { groupe(17), seconde(11), temps(28), boire(6) }\end{array}$ \\
\hline $\begin{array}{l}\text { C. Politique et } \\
\text { institutions }\end{array}$ & $\begin{array}{l}\text { élu(28), avis(29), commission(24), député(19), parlement(49), peuple(25), } \\
\text { pouvoir(91), referendum(24), région(15), représentant(16), rôle(16), vote(50), } \\
\text { représenter(23), voter(37), conseil(19), constitution(35), décision(23), } \\
\text { démocratie(31), gouvernement(23), décide(15), global(15), citoyen(20), nation(11), } \\
\text { belge(27), législatif(6), manière(14), national(28), initiative(6), intérêt(22), } \\
\text { personne(21) }\end{array}$ \\
\hline D. Religion & $\begin{array}{l}\text { avortement(6), chrétien(40), juif(7), mort(11), musulman(47), soumis(8), } \\
\text { spécifique(8), christianisme(7), culte(7), culture(24), église(12), empire(5), état(41), } \\
\text { femme(32), peine(11), prêtre(7), valeur(17), viande(7), voile(12), confondre(8), }\end{array}$ \\
lapider(5), adultère(7), catholique(28), islam(43), laïcité(28), référence(9), \\
religieux(85), judéo(26), laïc(21), grec(6), extrémisme(7), remonter(5), expressif(4), \\
liberté(8), mari(6)
\end{tabular}

Sans entrer dans le détail, soulignons que ces classes correspondent pour partie (et pour partie seulement) au scénario de la discussion, et ne reflètent pas simplement les différences entre les groupes. Seule la classe «Economie» ne correspond à aucune question en particulier. Alceste permet de confirmer le poids des questions économiques dans les

\footnotetext{
9 Nous avons choisi, après plusieurs tests, de mettre en variables supplémentaires (techniquement, en majuscules) les mots présents dans les questions affichées en face du groupe (par exemple, EUROPEEN/NES, NATIONS, ELU/S, EXPERTS, MARCHE, TURQUIE), de façon à éviter que la reprise de ces mots par les participants surdétermine la classification

${ }^{10}$ Les noms de classe ne sont pas générés par le logiciel, elles ont été labellisées par nos soins.
} 
discussions puisqu'elles représentent $40 \%$ de notre corpus (en tout cas de la partie classée par le logiciel qui représente ici plus de $66 \%$ des transcriptions).

Si l'on s'intéresse plus précisément à la question des évocations de pays dans ces discussions, on observe que certains d'entre eux se retrouvent associés à d'autres univers sémantiques que la classe «Nations ». Ainsi, la Belgique est significativement associée aux mots de la classe «Politique et institutions ", renvoyant à l'idée que le système politique belge permet à ses citoyens, par analogie mais aussi et surtout par familiarité, de s'approprier ou à tout le moins d'appréhender le système européen dans sa complexité. Par ailleurs, la Chine et la Pologne apparaissent quant à elles dans la classe "Economie », ainsi que, quoique de manière moins significative, le Congo, l'Inde ou la Tchéquie. Cette classe «Economie » comprend également la présence significative de certains pays d'Europe de l'Ouest dont la France, la Suisse ou encore le Luxembourg ${ }^{11}$.

Que dire maintenant de ce qui nous intéresse ici, à savoir la classe « Nations »?

Tout d'abord, il s'agit de la seconde classe la plus importante en taille $-16,6 \%$ des unités de texte classées. Ensuite, si on se réfère au tableau ci-dessous reprenant l'ensemble des formes caractéristiques de la classe ${ }^{12}$, on note qu'elle rassemble une part importante des noms de pays évoqués par les participants, qu'ils soient européens - Angleterre, Yougoslavie, Allemagne, Portugal et Espagne - ou non - Etats-Unis, Russie, ainsi que certains pays africains et asiatiques. Ces mots sont d'abord évoqués en relation avec le vocabulaire de la comparaison : ce qui est différent ou semblable, ce qui est plus ou moins, proche ou lointain, etc. De plus, une part importante de l'univers sémantique que constitue la classe relève des questions géostratégiques. A noter également la place faite à l'élargissement mais aussi aux aspects de temporalité. Enfin, la classe «Nations» comporte bien le vocabulaire des sentiments.

Tableau 2 : Ensemble des formes caractéristiques de la classe « Nations »

\begin{tabular}{|l|l|}
\hline $\begin{array}{l}\text { Pays } \\
\text { européens }\end{array}$ & $\begin{array}{l}\text { anglais(18), Yougoslavie(9), Allemagne(17), Portugal(7), Angleterre(11), } \\
\text { Espagne(14), EUROPE(164), EUROPEENNE(72), EUROPEENS(32) }\end{array}$ \\
\hline $\begin{array}{l}\text { Pays non } \\
\text { européens }\end{array}$ & $\begin{array}{l}\text { américains(21), Amérique(16), Etats-Unis(32), Tunisie(8), asiatique(8), Russie(11), } \\
\text { russe(5), Afrique (18), Maroc(8), latine(5), TURQUIE(45) }\end{array}$ \\
\hline Comparaison & $\begin{array}{l}\text { différent(50), bas(20), manque(7),haut(12), positif(8), totalement(8), diversité(5), } \\
\text { priorité(5), objectif(8), grand(28), fort(9), plus(114), très(41), entre(32), par } \\
\text { rapport(44), autre(71), loin(8), au-delà de(4) }\end{array}$ \\
\hline Commun & $\begin{array}{l}\text { union(40), commun(12), né(8), uni(4), commune(9), social(27), comme(92), } \\
\text { interne(5), proche(6), proche de (2), }\end{array}$ \\
\hline Elargissement & rentrer(25), élargir(7), membre(10, nouveau(15) \\
\hline Affect & sentir(20), sentiment(10), sens(33) \\
\hline Géostratégie & $\begin{array}{l}\text { pays(134), géographie(8), continent(7), lieu(6), nationalisme(5), militaire(12), } \\
\text { guerre(46), arme(13), conflit(4), puissance(14), géostratégique(4), économie(75), } \\
\text { monnaie(13), bloc(7), histoire(33), partie(39), catastrophe(6), chimère(6), }\end{array}$ \\
\hline Temporalité & aujourd'hui(44), lendemain(3), à présent(2), déjà(36), depuis(12) \\
\hline Autres & vers(14), me(40), suis(60) \\
\hline
\end{tabular}

Résumons les enseignements de cette exploration automatique du corpus. Tout d'abord, l'analyse Alceste atteste d'une présence non négligeable d'un univers sémantique lié à la géostratégie au sein duquel les Européens de l'Ouest sont évoqués, dans leurs relations

\footnotetext{
11 Tous les noms de pays n'apparaissent pas dans le tableau 1 car ils ne sont pas parmi les mots les plus significatifs de la classe. Ils arrivent plus loin dans la liste complète des mots associés que nous ne pourront pas reproduire dans cet article, faute de place

${ }^{12}$ Les formes caractéristiques illustratives apparaissent en gras ; en minuscules, les mots les plus usuels, ou mots outils ; en majuscules, les mots que nous avons choisi de mettre en variables supplémentaires.
} 
avec les Américains notamment; cet univers est marqué par des émotions. Par ailleurs, l'importance de la catégorie «Economie » et la présence à la fois de la Chine et la Pologne au sein de celle-ci et la relative absence des Européens de l'Ouest confortent notre interprétation de départ qui consistait à affirmer l'importance de ces pays «hors Europe » et de l'aspect économique dans la compréhension des dynamiques de nos discussions portant sur l'Europe.

Enfin, si il y a une spécificité de l'univers sémantique duquel relève les Européens de l'Ouest, celle-ci se trouve dans la comparaison ou la différentiation, tandis que la Belgique et la France, à savoir les communautés nationales, se situent dans d'autres univers sémantiques. En effet, il apparait que lorsque nos participants mobilisent leur communauté nationale en la nommant précisément ${ }^{13}$, ce n'est pas généralement pour la comparer aux Européens de l'Ouest ou pour l'opposer aux Etats-Unis mais pour, dans le cas des Belges (francophones), la situer dans le système institutionnel et politique européen ou, dans le cas français, en lien avec la globalisation de l'économie.

\section{Les Européens de l'Ouest, entre indifférence et neutralité.}

Pour approfondir ce que les Belges (francophones) et les Français disent de leurs voisins, nous avons ensuite procédé à une codification des séquences de discussion comportant l'évocation de pays ${ }^{14}$, à l'aide d'un CAQDAS (Computer Assisted Qualitative Data Analysis). Inspiré de la Grounded theory (Glaser \& Strauss, 1967), au sens où la codification est un outil de construction des concepts fondamentaux de l'analyse, Atlas.ti peut aussi être utilisé pour compter/mesurer la présence de thèmes dans un texte, ainsi que les relations que ces thèmes occupent entre eux. C'est ainsi que nous avons repéré, grâce à une recherche automatique, toutes les séquences comportant les noms de pays, de leurs habitants ou les adjectifs correspondant et les avons codés. Comme le montre le tableau 3 ci-dessous, ce premier comptage confirme la présence quantitative des Européens de l'Ouest dans les discussions.

Tableau 3 : Présence des noms de pays ou de nationaux ou d'adjectifs nationaux Effectifs (Quotes)

\begin{tabular}{|l|c|c|}
\hline Pays & Effectifs & \% \\
\hline Nation & 355 & 33 \\
Belgique & 172 & 16 \\
France & 183 & 17 \\
\hline Europe & 440 & 40 \\
France (mobilisée par les Belges) & 78 & 7 \\
Pays d'Europe de l'Ouest & 272 & 25 \\
Pays d'Europe de l'Est & 90 & 8 \\
\hline Reste du monde & 292 & 27 \\
Pays d'Afrique & 63 & 6 \\
Pays d'Asie & 85 & 8 \\
Etats-Unis & 105 & 10 \\
Autres (dont Russie) & 39 & 3 \\
\hline Total & 1087 & 100 \\
\hline
\end{tabular}

\footnotetext{
${ }^{13}$ Le plus souvent, l'évocation de la communauté nationale est implicite : en parlant de ce qui se passe autour d'eux, les participants ne nomment pas nécessairement la France ou la Belgique. Ces évocations implicites ne sont bien entendues pas prises en compte par une analyse de contenu comme celle-ci. A contrario, on peut considérer qu'une énonciation explicite de leur communauté nationale n'est pas sans signification.

${ }^{14}$ A l'exception de la Turquie dont l'évocation n'est pas seulement spontanée puisqu'une question porte spécifiquement sur son adhésion à l’Union.
} 
En effet, $40 \%$ des mentions explicites faites à des nationalités renvoient à des pays Européens, et près des deux tiers de ces références portent sur des pays d'Europe de l'Ouest. Cette part est cependant un peu gonflée par l'importance des références que les participants belges font à la France, qui est en partie due à l'animation française ; elle est à l'inverse un peu sous-estimée du fait d'une tendance à la co-évocation de plusieurs pays européens dans un même fragment de discussion (comptés une seule fois dans le tableau). Cette forte présence des mots désignant les Européens de l'Ouest contraste avec le peu d'importance que, d'après notre interprétation de la dynamique de chaque groupe, les participants nous semblent leur avoir accordée. Pourquoi ?

Une façon d'expliquer notre "surdité » première sur le thème des Européens, particulièrement de l'Ouest, serait que les séquences où ceux-ci sont évoqués correspondent rarement à des «moments sensibles ", sur lesquels se base principalement l'analyse interprétative (Kitzinger \& Farquhar, 1999). Or ce repérage par les «moments sensibles» n'est pas sans rapport avec l'objet de ce texte puisqu'il s'agit à l'évidence de moments qui mettent en jeu des émotions, les participants s'abandonnant et abandonnant par là même le registre cognitif et/ou distancié. Les comptages effectués via Alceste ou via Atlas.ti ne nous informent en rien sur la teneur affective des propos échangés - sinon que l'analyse Alceste indique la présence d'un vocabulaire lié aux sentiments. Or la question qui est posée ici n'est pas tant ce que pensent ou disent les Européens les uns des autres que ce qu'ils ressentent les uns pour les autres. Il a donc fallu affiner la codification et tenter d'évaluer la teneur affective des propos tenus.

Nous avons donc appliqué, à toutes les séquences évoquant directement un nom de pays, d'habitant, ou un adjectif de nationalité, un codage émotion positive / émotion négative / indifférence, en faisant bien la part entre la tonalité émotionnelle de la séquence et son rapport avec le pays évoqué ${ }^{15}$. Cela n'a pas été sans mal.

Tableau 4 : Emotions et indifférence

\begin{tabular}{|l|c|c|c|c|}
\hline Pays & $\begin{array}{c}\text { Emotions } \\
\text { (effectifs) }\end{array}$ & $\begin{array}{c}\text { Indifférence } \\
\text { (effectifs) }\end{array}$ & $\begin{array}{c}\text { Total } \\
\text { émotions }\end{array}$ \\
\hline Nation & 154 & 181 & 335 & $\mathbf{4 6}$ \\
France & 68 & 89 & 157 & $\mathbf{4 3}$ \\
\hline Europe & 86 & 92 & 178 & $\mathbf{4 8}$ \\
France (mobilisée par la Belgique) & 198 & 171 & 369 & $\mathbf{5 4}$ \\
Pays d'Europe de l'Ouest & 45 & 33 & 78 & $\mathbf{6 0}$ \\
Pays d'Europe de l'Est & 104 & 108 & 212 & $\mathbf{4 9}$ \\
\hline Reste du monde & 49 & 30 & 79 & $\mathbf{6 2}$ \\
Pays d'Afrique & 182 & 85 & 267 & $\mathbf{6 8}$ \\
Pays d'Asie & 36 & 28 & 63 & $\mathbf{5 7}$ \\
Etats-Unis & 50 & 26 & 76 & $\mathbf{6 6}$ \\
Autres & 73 & 23 & 96 & $\mathbf{7 6}$ \\
\hline Total & 23 & 8 & 31 & $\mathbf{7 4}$ \\
\hline
\end{tabular}

\footnotetext{
${ }^{15}$ On ne pourra pas développer ici toute la difficulté - bien soulignée par ce spécialiste de l'analyse des émotions en politique, Philippe Braud (Braud, 1996) - qu'il peut y avoir à coder des émotions, du fait notamment que coder nécessite de décontextualiser des segments de discours, mais aussi de par les ambivalences qui marquent en permanence ce type de discussions.

${ }^{16}$ Le total des citations codées - 971 - est inférieur à l'ensemble des séquences repérées dans le tableau précédent qui comportent des noms de pays car un certain nombre d'entre elles sont soit incompréhensibles, soit constituent des séquences « écho », de reprise destinées à assurer une bonne compréhension du propos.
} 
Avec toutes les réserves nécessaires ${ }^{17}$, ce décompte permet bien de faire apparaître une certaine spécificité des évocations des Européens dans les entretiens. Ils sont proches des évocations des situations nationales - dans lesquelles le pays est souvent une façon de situer ce dont on parle et n'est qu'indirectement l'objet de la discussion - et diffèrent des évocations du reste du monde, plus nettement chargées émotionnellement. On notera que la charge affective qui caractérise la France évoquée par les Belges peut de nouveau être comprise en rapport à la difficulté, pour les participants, d'évoquer le pays des animatrices. Quant aux Européens de l'Est, on voit qu'ils sont, en termes de charge affective, plus proches du reste du monde que des Européens de l'Ouest.

On observera ici que cette répartition des émotions - d'autant plus fréquente que la zone évoquée est éloignée des locuteurs - peut sembler contrevenir à l'idée répandue selon laquelle seuls les sujets proches de l'expérience ordinaire des sujets sont réellement investis de sens. Or ici, ces évocations sont spontanées et non suscitées par le questionnement des chercheurs. Sans doute est-ce pour cela que les pays lointains sont évoqués surtout dans un rapport chargé d'émotion car ils ne surgissent pas, à l'inverse du contexte national, «naturellement » dans la conversation. On notera d'ailleurs que ces évocations sont souvent entachées de méconnaissance et d'erreurs manifestes.

Tableau 5 : Emotions positives et négatives

\begin{tabular}{|l|c|c|c|c|}
\hline Pays & $\begin{array}{c}\text { Emotions } \\
\text { positives }\end{array}$ & $\begin{array}{c}\text { Emotions } \\
\text { négatives }\end{array}$ & Total & $\begin{array}{c}\text { \% Emotions } \\
\text { positives }\end{array}$ \\
\hline Nation & 64 & 99 & 163 & $\mathbf{3 9}$ \\
Belgique & 29 & 45 & 74 & $\mathbf{3 9}$ \\
France & 35 & 54 & 89 & $\mathbf{3 9}$ \\
\hline Europe & 99 & 108 & 207 & $\mathbf{4 8}$ \\
France (mobilisée par la Belgique) & 21 & 25 & 46 & $\mathbf{4 6}$ \\
Pays d'Europe de l'Ouest & 55 & 55 & 110 & $\mathbf{5 0}$ \\
Pays d'Europe de l'Est & 23 & 28 & 51 & $\mathbf{4 5}$ \\
\hline Reste du monde & 62 & 121 & 183 & $\mathbf{3 4}$ \\
Pays d'Afrique & 25 & 11 & 36 & $\mathbf{6 9}$ \\
Pays d'Asie & 13 & 37 & 50 & $\mathbf{2 6}$ \\
Etats-Unis & 15 & 61 & 76 & $\mathbf{1 9}$ \\
Autres & 9 & 12 & 21 & $\mathbf{4 3}$ \\
\hline Total & 224 & 328 & 552 & $\mathbf{4 0}$ \\
\hline
\end{tabular}

Lorsqu'on regarde plus en détails la répartition entre affect positif et affect négatif, l'Europe de l'Ouest se distingue des autres parties du monde - y compris, cette fois, des pays nationaux - par une forme de neutralité, au sens d'un équilibre entre les émotions positives et négatives qui lui sont attachées. Ceci la distingue du bilan globalement négatif des affects que les participants attachent à l'évocation de la situation dans leur nation, et des émotions contrastées qui caractérisent les relations qu'ils entretiennent avec le reste du monde - même si la différence avec les Européens de l'Est semble moins forte que ne le laissait supposer l'analyse interprétative. Pour mieux comprendre ce bilan, revenons, à travers des exemples, sur le contenu des séquences de discussions portant sur ces différents pays.

\footnotetext{
${ }^{17}$ Portant sur le caractère toujours discutable d'un tel codage d'abord, mais aussi sur l'absence de test de significativité des différences de pourcentage d'affects entre les différents pays ou groupes de pays. Ce qui nous semble significatif, au sens en tous cas où on peut l'investir de sens, c'est la structure de ces différences, et la façon dont les résultats dans le cadre national se distinguent du reste du monde, l'Europe occupant une position intermédiaire.
} 


\title{
Différences relatives, différences radicales : questions sur la souveraineté
}

Selon la théorie de l'in- et out-group qui sous-tend de nombreux travaux sur l'identité nationale, tout particulièrement dans son rapport avec l'identité européenne ${ }^{18}$, le groupe national est construit dans la réification de différences, produit des idéologies nationales mais relayée dans l'expérience, plus ou moins fantasmée, que chacun a des « autres ». Aussi avonsnous voulu aller regarder de plus près la façon dont, comparativement, sont évoquées les différences que les participants français et belges (francophones) de nos groupes se reconnaissent par rapport aux autres pays, et notamment les Européens de l'Ouest. On peut distinguer grossièrement deux modes de traitement de la différence : de l'ordre du relativisme emprunt d'une forme de valorisation dans le cas des Européens de l'Ouest; de l'ordre du radical, voire de l'antinomie, dans le cas du reste du monde - lequel inclut alors, à bien des égards, les Européens de l'Est.

Du côté de l'Europe de l'Ouest donc, ce qui revient le plus souvent, c'est d'abord la reconnaissance de la persistance des différences entre les nations, différences fondées sur des histoires respectives longues et riches, et donc valorisées; pourtant, ces différences sont considérées comme surmontées au sens où elles n'empêchent pas que vivent ensemble dans l'Union Européenne, et sans perte réelle pour les uns et les autres, des peuples très différents. L'extrait ci-dessous intervient à la fin du groupe militant à propos de la question de la Turquie. Maxime a 25 ans. Licencié en communication, il est permanent chez Ecolo, et se situe lui-même en position 2 sur l'axe gauche-droite en 10 positions. D'origine maghrébine, mais peu visible, il a souhaité utiliser un nom d'emprunt pour ne pas la dévoiler. Il se retrouve, en fin de discussion (Extrait 1) au cœur d'un conflit d'autant plus intense qu'inattendu, en réagissant fermement aux propos quasi racistes tenus par certains participants.

\begin{abstract}
Extrait 1 (Militants, Bruxelles)
Maxime : Pourquoi ça poserait spécialement un problème [l'intégration de la Turquie], sur quelle base, c'est une peur ça c'est une crainte c'est mais qu'est-ce qu'elle a d'objectif enfin (remarque inaudible d'un autre participant) qu'est-ce qui nous permet de dire qu'elle va poser problème alors qu'on sait que l'Europe a (hésitant) déjà réussi à surmonter des tas de différences, en s'en sortant enfin je pense assez (hésitant) enfin pas mal quoi ne fut ce que... enfin je sais pas est-ce : est-ce qu'il y a 30 ans on nous aurait dit que dans le même, dans le même espace on aurait réussi à avoir des gens aussi différents que des Irlandais et des Grecs?
\end{abstract}

Cette façon de mettre au même niveau l'Irlandais et le Grec, par delà leurs différences, et sans qu'aucune préférence ou proximité n'apparaisse, caractérise bien cette forme de reconnaissance minorée et valorisée des différences entre Européens de l'Ouest qu'on trouve dans les discussions. Bien sûr, elle est loin d'être unanime, et est tout particulièrement le fait des participants appartenant à des minorités ethniques ${ }^{19}$, mais pas seulement. La façon dont David, 24 ans, sergent, proche du PS, intervient dans une discussion sur les bienfaits (ou non) d'une unification de l'enseignement supérieur au niveau européen (Extrait 2), et oppose « des pays là-bas à l'Est » avec «nos pays » confirme bien la relativité des différences que les participants à nos groupes reconnaissent entre les pays d'Europe de l'Ouest.

\section{Extrait 2 (Employés, Bruxelles)}

David = ben déjà on a quand même des, des pays qui sont je vais pas dire en voie de développement mais on a des pays qui sont beaucoup moins, qui ont beaucoup moins accès que nous, que nos pays à, à

\footnotetext{
${ }^{18}$ Voir, pour des exemples de l'importation des théories psycho-sociales dans ce domaine (Hermann, Risse \& Brewer, 2004 ; Bruter, 2005). Pour une discussion des limites de cette importation (Duchesne, à paraître, 2008).

${ }^{19}$ Nous avons pris un soin particulier à recruter des participants appartenants aux minorités ethniques des deux pays, et avons pu observer un effet important de leur présence dans les discussions.
} 
l'information à (hésite) de bonnes écoles donc des chercheurs enfin il y a des différences quoi d'office (pause). Allez je vais pas, je vais pas commencer à dire que si tu vas, si tu vas suivre des cours en Pologne tu vas être plus abruti qu'un autre mais je suppose qu'il y a quand même plus de spécialités ben au niveau de la médecine dans nos pays que dans des pays là-bas à l'Est.

Autrement dit, avec des inflexions, c'est quand même l'idée de la relativité de ces différences qui domine dans ces groupes, en même temps que leur valorisation. On note d'ailleurs l'absence des stéréotypes attendus sur les Allemands, les Italiens, etc. Cette relativisation se traduit par la faiblesse relative des affects attachés à chacun des pays d'Europe de l'Ouest.

A l'inverse, les autres parties du monde, à commencer par l'Europe de l'Est, sont le plus souvent évoquées comme radicalement différentes. Les Etats-Unis sont le contre modèle, celui qui fonde la relativité des différences entre Européens comme l'illustre l'extrait 3. Lorsque les participants de ce groupe ouvrier parisien y évoquent la question de la justice, ils associent la France à l'Allemagne, et par extension les autres pays d'Europe de l'Ouest, en les opposant aux Etats-Unis. Cette opposition leur permet d'ailleurs de contourner une absence évidente de connaissance quant à la réalité des systèmes de justice dans les différents pays :

\section{Extrait $3^{20}$ (Ouvriers, Paris)}

Albert (à l'animatrice) = ben ce que je dis c'est vrai que l'Europe a un certaine justice que d'autres pays ont pas forcément :

Animatrice : d'accord :

Albert : souvent la justice européenne ressemble plus : c'est-à-dire que les Allemands ressemblent plus aux Français que les : les Américains par exemple (Habiba approuve) ils ont une justice complètement différente (Habiba l'approuve, Albert poursuit, vers l'animatrice) eux ils ont la peine mort que l'Europe je crois dans toute l'Europe la peine de mort est (hésitant) abolie je crois (hésite encore) y'a pas ce genre : (Ghislaine appuie) enfin ce genre de choses je veux dire.

La différence radicale est connotée généralement négativement dans le cas des EtatsUnis, de la Chine et de l'Europe de l'Est. Elle emprunte tour à tour aux registres de la géopolitique en général et de l'économie mondiale en particulier, et dénote, par le double jeu de la politique et de la puissance à l'échelle du monde, la crainte de se retrouver dans le flot de la mondialisation de l'économie. Continuons avec le groupe d' «ouvriers » parisiens, particulièrement animé. Deux femmes, originaires d'Afrique du Nord, s'opposent, sur un mode haut en couleurs, sur les principes et les conditions de l'intégration républicaine. Les autres assistent à leur conflit, tantôt amusés, tantôt médusés. Tous cependant partagent une sorte de fatalisme, de sentiment d'impuissance à l'égard d'un monde dominé économiquement par les grandes puissances, les Etats-Unis bien sûr, mais aussi la Chine, voire l'Inde, et dans lequel l'Europe ne pèse guère.

\section{Extrait 4 (Ouvriers, Paris)}

Lionel : donc on a beau (hésite) ouvrir enfin, l'Europe... l'agrandir, pour essayer de (hésite) enfin, l'idée c'est de défendre les gens qui habitent en Europe en ouvrant l'Europe

Albert (devant lui, le coupant) : c'est pour ça que ça s'appelle ....

Lionel (poursuit) : c'est protéger les Européens (mime l'emprise) de qui ?

Albert : ça va plus s'appeler l'Europe

\footnotetext{
${ }^{20}$ Les extraits 3 et 4 mettent en scène un groupe d'ouvriers-classe populaire parisien qui rassemble : Albert, 42 ans, diplôme de niveau de $3^{\text {ème }}$, actuellement au chômage, qui se positionne en 5 ou 6 sur l'échelle gauchedroite ; Ghislaine, d'origine antillaise, 26 ans, aide-soignante, position 4; Geoffroy, 33 ans, ouvrier dans le secteur de l'imprimerie, centriste ; Lionel, 42 ans, agent de sécurité, ne se situe pas sur l'échelle gauche-droite mais déclare avoir voté Besancenot lors du dernier scrutin; Yasmina, 35 ans, d'origine maghrébine, femme au foyer en situation précaire, n'a pas voté aux dernières élections mais se positionnerait plutôt à gauche ; Habiba, 41 ans, elle aussi d'origine maghrébine et femme au foyer mais femme de commerçant, se positionne en 3 ou 4 sur l'échelle gauche-droite.
} 


\begin{abstract}
Yasmina : oui
Lionel (toujours à Yasmina) : on a peur, des Chinois on a peur des Indiens :

Geoffroy : on a peur en fait des Etats-Unis donc :

Lionel : (coupant Geoffroy) parce que là ils, mais ils travaillent pour nous :

Albert (de biais, coupant Geoffroy) je pense que c'est plutôt les Etats-Unis

Lionel : mais en même temps

Albert : c'est eux qui décident en po....

Ghislaine (vers Albert, lui coupant la parole) c'est eux qui décident (Yasmina approuve)

Geoffroy (devant lui) : ouais voilà donc, on veut les contrer et

Habiba (devant elle) : ben oui

Albert : c'est eux les barbares de la finance

Geoffroy (à part lui) : ouais ouais

Yasmina (vers Albert) : exactement c'est eux qui ont tout le pouvoir on dirait

Albert : c'est eux :

Lionel (vers les autres) : mais les Etats-Unis sont en train de, ils ont plus tout le pouvoir

Yasmina (vers les autres) : voilà

Albert (devant lui) : c'est eux qui tiennent l'économie hein

Lionel : il faut pas oub ( $s$ 'interrompt) ils tiennent l'économie mais pas toute l'économie parce que la Chine (Yasmina approuve) à l'heure actuelle commence à peser mais les Etats-Unis à côté ils vont (hésite) d'ici deux ou trois ans c'est fini
\end{abstract}

Dans ce groupe, par ailleurs si divisé, tous se retrouvent avec véhémence dans ce sentiment de débordement par une réalité qui se joue ailleurs et échappe à ce qu'ils connaissent, là où ils se reconnaissent, à savoir les frontières du groupe national d'abord, mais étendu ici aux frontières de l'Europe. Autrement dit, derrière le registre de la différence radicale, c'est celui de la souveraineté - et le plus souvent, de la souveraineté en péril - qui s'exprime. Comme l'a montré Benedict Anderson, la caractéristique de la communauté nationale est d'être imaginée «comme intrinsèquement limitée et souveraine» (Anderson, 1996, 19). Si les Européens de l'Ouest sont bien reconnus par tous, dans ces groupes, comme faisant maintenant partie de leur communauté de destin, celle-ci n'a à l'évidence pas encore fait la preuve de sa souveraineté. Les Européens de l'Ouest n'apparaissent donc pas (ou plus) vraiment comme des concurrents, susceptibles de donner prise à des émotions négatives ${ }^{21}$, sans pour autant que se profile, à l'échelle européenne, une communauté imaginée comme souveraine qui les engloberaient tous.

\title{
Conclusion :
}

Revenons sur la notion «d'angle mort » que nous avons utilisée dans la formulation de notre hypothèse de départ pour qualifier l'espace que les Européens de l'Ouest occupent dans ces discussions sur l'Europe : certes ils sont bien là, mais l'intensité émotionnelle des échanges qui portent sur eux est faible, et par comparaison avec les différences que nos participants marquent avec le reste du monde, celles-là sont largement relativisées. Autrement dit, il semble qu'à la faveur du processus d'intégration, et/ou dans la dynamique de la globalisation, les Européens de l'Ouest ont perdu pour les Belges (francophones) et les Français, le statut «d'autres », au sens de ceux qui fondent l'identité de soi par la différence, mais surtout, ceux qui marquent les frontières de la souveraineté nationale. Ce n'est donc pas le désamour qui freine aujourd'hui la construction d'une communauté politique à l'échelon européen, du moins vu du côté des Français et des Belges (francophones). Et ces discussions ne permettent pas de nourrir la thèse du développement d'une "préférence continentale »

\footnotetext{
${ }^{21}$ On lit par contre très clairement, dans les groupes anglais, la persistance d'un conflit latent de souveraineté avec les grandes nations européennes continentales, notamment les Français et les Allemands.
} 
propre à compenser le déficit d'attachement des européens pour leur Union. Ce résultat (provisoire $^{22}$ ) peut être interprété de différentes façons.

Les tenants du post-nationalisme y liront les premiers signes de la construction d'une communauté politique d'un genre nouveau, fondée sur l'acceptation de la diversité et tournant le dos à l'intégration culturelle (Nikolaïdis, 2004). A l'inverse, ceux qui mettent la construction européenne en perspective avec le long processus qui a marqué la construction des nations pourront y voir l'effet d'un travail en cours, une communauté « en progrès », dans laquelle les différences subsistent mais commencent à être considérées comme une richesse, et non plus comme une menace (Delmotte, 2007b).

Mais ce résultat peut aussi nous interroger sur la pertinence de l'échelon continental dans le bouleversement des frontières nationales mis en jeu par la construction européenne, et plus largement, par la globalisation. Même si nos participants français et belges (francophones) évoquent fréquemment leurs voisins européens, en creux, c'est surtout leur nation dont ils discutent la perte de substance dans le courant de la globalisation. Et l'Europe y apparaît rarement, dans les groupes populaires et intermédiaires en tous cas, comme un échelon signifiant, comme un espace souverain. Est-ce parce qu'il est trop tôt pour observer les traces, qu'elles soient verticales ou horizontales, d'une communauté politique européenne en construction? Ou parce que ce que ce n'est pas cette histoire-là qui est en train de s'écrire ? Cela, notre enquête ne permet pas plus que les autres de le dire.

\section{Bibliographie}

Anderson Benedict (1996, éd. anglaise 1983), L’imaginaire national. Réflexion sur l'origine et l'essor du nationalisme, Paris, La Découverte.

Braud Philippe (1996), L'émotion en politique : problèmes d'analyse, Paris, Presses de Sciences Po.

Brugidou Matthieu (2008), L'opinion et ses publics. Une approche pragmatiste de l'Opinion publique, Presses de Sciences Po.

Bruter Michael (2005), Citizens of Europe? The Emergence of a Mass European Identity, Basingstoke, Palgrave.

Citrin Jack \& Sides John (2004), « Can Europe exist without Europeans ? Problems of Identity in a Multinational Community » in Hermann Margaret, Advances in Political Psychology vol.1, New York, Pergamon Press, p.4170 .

Delmotte Florence (2007), Le cour a ses raisons... Les affects et la communauté politique au prisme d'une sociologie historique, communication au deuxième colloque de la SEE/AFSP, Grenoble, 6/7 décembre.

Delmotte Florence (2007b), «Les résistances à l'Europe au prisme de la sociologie historique de Norbert Elias », in Coman Ramona \& Lacroix Justine (Eds.), Les résistances à l'Europe. Cultures nationales, idéologies et stratégies d'acteurs, Bruxelles, Éditions de l’Université de Bruxelles, p.17-29.

Déloye, Yves (1996), Sociologie Politique, Paris, La Découverte, coll. Repères.

Déloye Yves (2006), «Introduction: Eléments pour une approche socio-historique de la construction européenne. Premier état des lieux » in Politique Européenne, 18, hiver 2006, p.5-16.

Duchesne Sophie (à paraître, 2008), «Waiting for a European Identity... Reflections on the process of identification with Europe », Perspectives on European Politics and Society, vol.9/4, December, 397-410.

Duchesne Sophie \& alii (2008), «Attention to Europe: where social groups really differ. Comparative analysis of French, British and (French speaking) Belgians focus groups", présentation au colloque «European citizenship revisited », Oxford, 23 juin,

\footnotetext{
${ }^{22}$ Une partie seulement des groupes que nous avons organisés a été analysée pour l'instant.
} 
http://erg.politics.ox.ac.uk/materials/european_citizenship/european_citizenship.asp.

Duchesne Sophie \& Frognier André-Paul (2008), «National and European Identifications : a Dual Relationship », Comparative European Politics, 6, p.143-168.

Duchesne Sophie \& Haegel Florence (2004), «La politisation des discussions, au croisement des logiques de spécialisation et de conflictualisation », Revue Française de Science Politique, 54 (6), décembre 2004, p. 877-909.

Duchesne Sophie \& Van Ingelgom Virginie (2008), «Comment les discussions deviennent politiques, lorsque des français, des Anglais ou des Belges francophones parlent de l'Europe? », Politique Européenne, Hiver 2008, 24, p. 145149.

Garcia Guillaume, Van Ingelgom Virginie \& alii (2008), «Comparing conflict on European issues », présentation au colloque « European citizenship revisited », Oxford, 23 juin,

http://erg.politics.ox.ac.uk/materials/european_citizenship/european_citizenship.asp.

Glaser Barney \& Strauss Anselm (1967), The discovery of grounded theory: strategies for qualitative research, Londres, Weidenfield and Nicolson.

Habermas Jürgen (2000), Après l'Etat-nation. Une nouvelle constellation politique, Paris, Fayard.

Haegel Florence \& alii (2008), "National Framing revisited: French, British and French speaking Belgian citizens arguing about Europe", présentation au colloque «European citizenship revisited », Oxford, 23 juin, http://erg.politics.ox.ac.uk/materials/european_citizenship/european_citizenship.asp.

Hamidi Camille (2006), «Éléments pour une approche interactionniste de la politisation. Engagement associatif et rapport au politique dans des associations locales issues de l'immigration », Revue Française de Science Politique, 51 (1), p. 5-26.

Hermann Richard K., Risse Thomas \& Brewer Marylinn B. (2004), Transnational Identities. Becoming European in the EU, Oxford, Rowman \& Littlefield publishers.

Kitzinger Jenny \& Farquhar Claire (1999), « The Analytical Potential of 'Sensitive Moments' in Focus Group Discussions » in Barbour Rosaline S. \& Kitzinger Jenny (Eds.), Developing Focus Group Research. Politics, Theory and Practice, London, Sage, p. 156-172.

Miller David (2002), Citizenship and National Identity, Cambridge, Polity Press.

Nikolaïdis Kalypso (2004), «We, the Peoples of Europe...», Foreign Affairs, November/December, p. 97-110.

Reinert Max (1993), «Les "mondes lexicaux" et leur "logique" à travers l'analyse statistique d'un corpus de récits de cauchemars », Langage et Société, 66, p. 5-39.

Sauger Nicolas, Brouard Sylvain \& Grossman Emiliano (2007), Les Français contre l'Europe ? Les sens du référendum du 29 mai 2005, Paris, Presses de Sciences Po.

Strudel Sylvie (dir.) (2002), Pratiques de la citoyenneté Européenne, Revue Internationale de Politique Comparée, 9 (1).

Soysal Yasemin (1994), Limits of Citizenship: Migrants and Postnational Membership in Europe, Chicago, University of Chicago Press.

Tajfel Henri (1982), Social Identity and Intergroup Relations, Cambridge, CUP. 